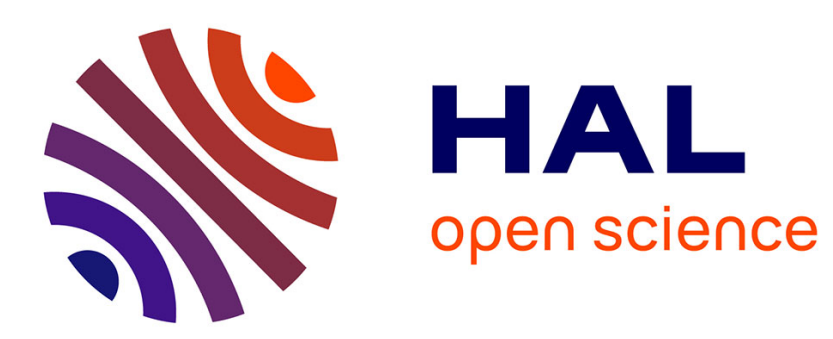

\title{
ADSORBED METAL LAYERS : STRUCTURE, WORK FUNCTION AND BONDING
}

E. Bauer

\section{To cite this version:}

E. Bauer. ADSORBED METAL LAYERS: STRUCTURE, WORK FUNCTION AND BONDING. Journal de Physique Colloques, 1977, 38 (C4), pp.C4-146-C4-154. 10.1051/jphyscol:1977423 . jpa00217139

\section{HAL Id: jpa-00217139 \\ https://hal.science/jpa-00217139}

Submitted on 1 Jan 1977

HAL is a multi-disciplinary open access archive for the deposit and dissemination of scientific research documents, whether they are published or not. The documents may come from teaching and research institutions in France or abroad, or from public or private research centers.
L'archive ouverte pluridisciplinaire HAL, est destinée au dépôt et à la diffusion de documents scientifiques de niveau recherche, publiés ou non, émanant des établissements d'enseignement et de recherche français ou étrangers, des laboratoires publics ou privés. 


\title{
ADSORBED METAL LAYERS : STRUCTURE, WORK FUNCTION AND BONDING
}

\author{
E. BAUER \\ Physikalisches Institut, Technische Universität Clausthal \\ and SFB 126, Clausthal-Göttingen, Germany
}

\begin{abstract}
Résumé. - On analyse les données publiées concernant la structure, le travail de sortie et les paramètres d'adsorption-désorption de métaux sur la surface (110) du tungstène, dans le but de déterminer les règles générales qui conditionnent ces quantités, en se basant sur des considérations élémentaires d'électronégativité et de rugosité atomique.
\end{abstract}

\begin{abstract}
The available data on the structure, work function and adsorption-desorption parameters of metals on $\mathrm{W}\{110\}$ surfaces are analyzed with the goal of obtaining general rules which determine these quantities, based on elementary electronegativity and atomic roughness considerations.
\end{abstract}

1. Introduction. - Adsorbate-substrate and adsorbate-adsorbate interactions determine the structure, the energetics, kinetics and the surface potential of adsorption layers. In spite of a considerable effort, a reliable quantum mechanical description of metal-metal adsorbate-substrate systems as a function of coverage still is not available. Therefore semi-classical calculations e.g. based on pairwise interaction potentials, or simple models, e.g. the Smoluchowski or the Topping model, have to be relied on. Predictions based on these calculations and models are risky. Therefore experimental evidence has to be used to formulate the rules which govern the adsorption of metals on metal surfaces and the relations between structure, energetics and surface potential. Such data are accumulating at a considerable rate, in particular for adsorption on W surfaces, especially on the $\{110\}$ and on the $\{100\}$ surface. Adsorption on the $\{100\}$ surface is rather complex due to its inherent temperature-dependent atomic roughness [1-3] and its instability upon heating in the presence of adsorption layers $[2,4,5]$. Therefore this paper will be concerned only with adsorption on the $W\{110\}$ surface which is the most stable crystal surface.

In section 2 the available experimental data and some models are reviewed. From these data rules are derived in section 3 which govern structure, surface potential and energetics as a function of coverage and the interrelation between these quantities. Section 4 gives a brief summary.

2. Experimental data. - Only those results will be discussed here for which structural data and work function change $(\Delta \phi)$ measurements are available. This excludes all systems which have been studied by field emission or thermionic emission only. Table I lists the adsorbates studied to date together with their electronegativities $X$, atomic diameters $d$ and sublimation energies $E_{\mathrm{s}}$ as well as some quantities derived from them: atomic density in hexagonal close packing $N^{\text {hcp }}, E_{\mathrm{a}}{ }^{\prime}=\frac{1}{12}\left(E_{\mathrm{sa}}+E_{\mathrm{ss}}\right)$ and $\left(X_{\mathrm{a}}-X_{\mathrm{s}}\right)^{2}$ from which two quantities the heat of adsorption/atom according to Pauling $E_{\mathrm{a}}=\frac{1}{12}\left(E_{\mathrm{sa}}+E_{\mathrm{ss}}\right)+\left(X_{\mathrm{a}}-X_{\mathrm{s}}\right)^{2}$ may be obtained. This quantity is of only qualitative value because it is based on the assumption of bonding with only one substrate atom. However, it shows clearly the influence of the electronegativity difference on the heat of adsorption. The adsorbates are divided in two major groups (I and II) corresponding to systems with large $(\Delta X>1.5)$ and small $(\Delta X<1.5)$ electronegativity differences between adsorbate and substrate respectively. For many of the adsorbates only $\Delta \phi$ and LEED data have been published and the coverages of many of the LEED patterns are uncertain. Reliable heat of adsorption data are still scarce. The kind of information available at present will be illustrated with some extreme cases from the point of view of electronegativity (Cs, $\mathrm{Ba}, \mathrm{Pd}, \mathrm{Au}$ ) and of atomic size ( $\mathrm{Be}, \mathrm{Cu}, \mathrm{Ba}, \mathrm{Cs})$. The structure is conveniently described in terms of the unconventional rectangular unit mesh shown in figure 1 instead of the usual unit mesh.

2.1. STRUCTURE: ELECTRONEGATIVITY INFLUENCE. A LEED study of Cs on W(110) [6] cooled to sufficiently low temperatures $(77 \mathrm{~K})$ revealed a sequence of hexagonal structures which developed out of a diffuse ring observed at atomic 
TABLE I.

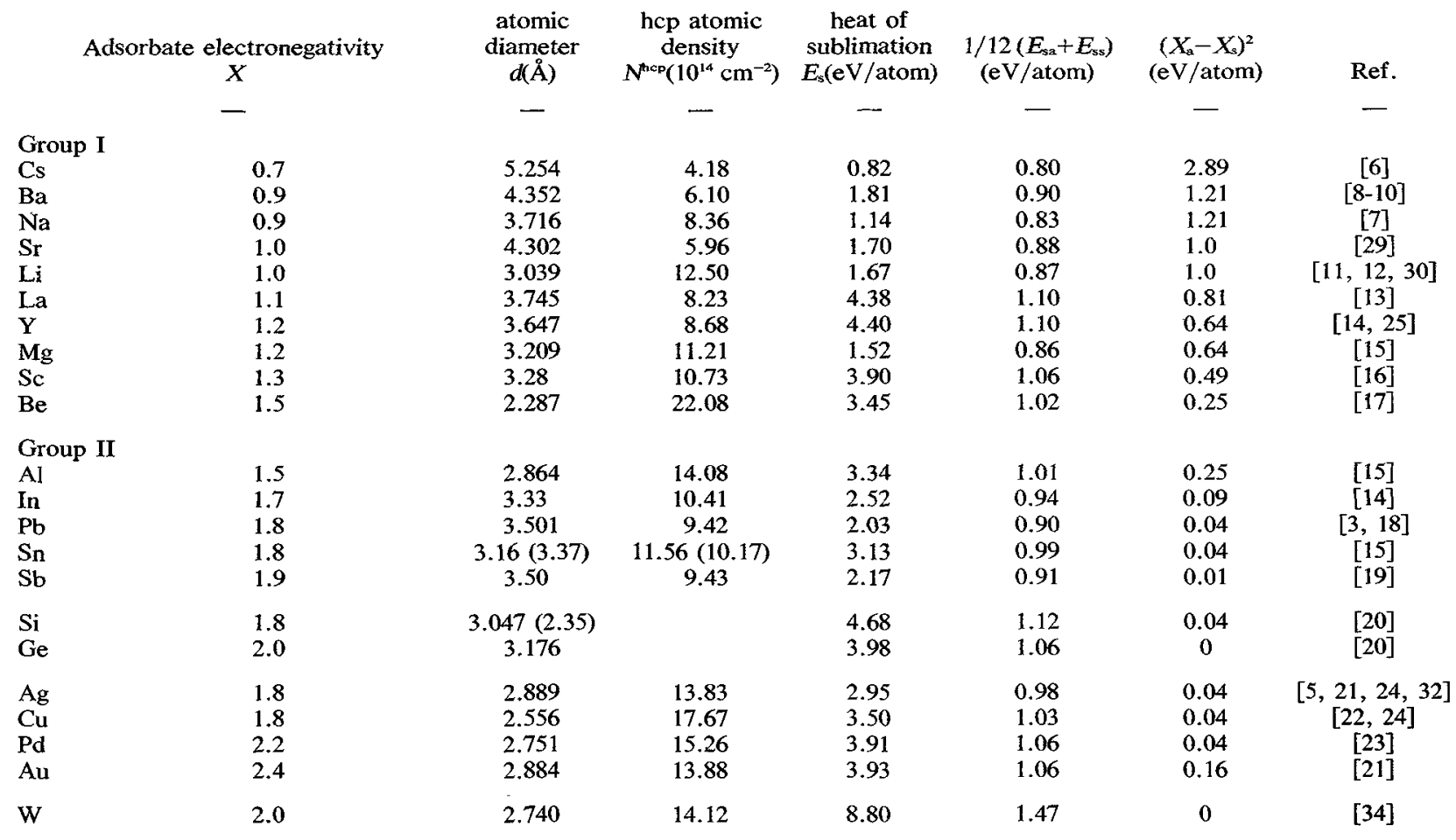

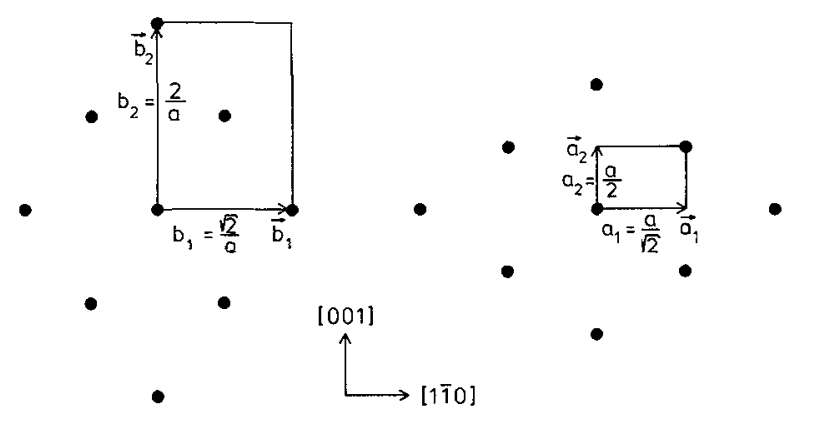

a)

b)

FIG. 1. - Unit mesh choices. a) reciprocal space, $b$ ) real space.

densities $N<2 \times 10^{14} \mathrm{~cm}^{-2}$. The interatomic distance decreased continously with coverage from $11 \AA$ to $4.7 \AA$, the only discontinuous feature being a sudden rotation of the hexagon by $90^{\circ}$ at the work function minimum. The work function decreased smoothly from $-5.3 \mathrm{eV}$ to minimum of $1.60 \mathrm{eV}$ at $N^{\min }=3.3 \times 10^{14} \mathrm{~cm}^{-2}$ and saturated at $2.1 \mathrm{eV}$ at $N^{\text {sat }}=5.2 \times 10^{14} \mathrm{~cm}^{-2}$ (coverage $\theta=1$ ). Beyond $\theta=1$, Cs crystals with hop structure grew. Ba shows a somewhat different behaviour [8]: here several discrete adsorbate structures with unit meshes of different shape form with increasing coverage. For each of these structures $\frac{d \phi}{d N}$ is constant, i.e. the dipole moment per adsorbed atom is changing linearely from the completion of one structure to the completion of the next. The transition from one structure to the next occurs either by continous compression - as in the case of Cs - or via a mixture of the two structures, each of which is retaining its interatomic distances. Also, similarily to $\mathrm{Cs}$, below the work function minimum at $N^{\min }=2.35 \times 10^{14} \mathrm{~cm}^{-2}\left(\phi_{\min }=1.7 \mathrm{eV}\right)$ one of the unit mesh axes is parallel to [001], above $\phi_{\min }$ parallel to $[110]$. Thus, in spite of differences in detail, the general features of $\mathrm{Cs}$ and $\mathrm{Ba}$ adsorption are very similar.

$\mathrm{Pd}$ and Au which differ from Cs and Ba not only in their electronegativity but also in their much higher heat of sublimation behave quite differently. Pd forms at room temperature a pseudomorphic layer which grows to a thickness of several monolayers upon further condensation [23]. At temperatures above $800 \mathrm{~K}$ only the first pseudomorphic monolayer is stable, the excess $\mathrm{Pd}$ forms three-dimensional crystals. Au [5] also forms a $(1 \times 1)$ structure at room temperature ; at elevated temperature a structure intermediate between the $\mathrm{W}(110)$ and the $\mathrm{Au}(111)$ plane results, with $N^{\text {sat }}=13.4 \times 10^{14} \mathrm{~cm}^{-2}$. Thicker layers consist of Au(111) layers compressed by $2.3 \%$. The respective LEED patterns and unit meshes are sketched in figure 2. No additional ordered structures are found below $\theta=1$. This could either be due to random adsorption or due to island formation. A distinction is in principle possible by LEED intensity measurements which have not been done, however. Thermal desorption spectroscopy also allows to distinguish between the two models on the basis of 
the desorption kinetics, but requires that the islands, if present, are still present at the desorption temperature. Thus a study of $\mathrm{Cu}[22]$ in the submonolayer range indicates that islands may be stable up to $1200 \mathrm{~K}$.

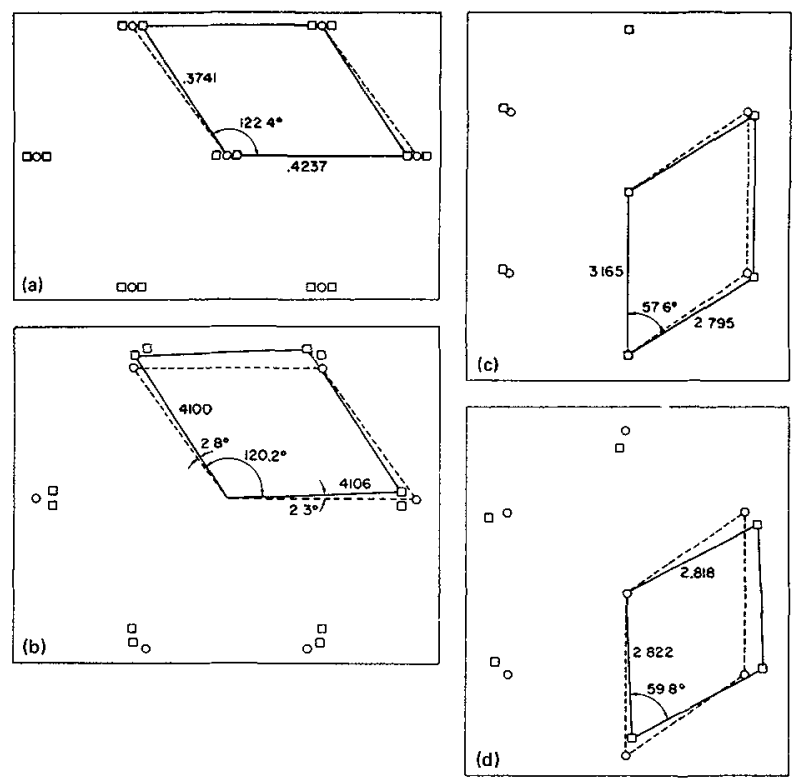

FIG. 2. - LEED patterns $(a, b)$ and unit meshes $(c, d)$ of $A u$ on W(110). $a, c) \theta \leq l, b, d) \theta>1$. Circles : W spots (a, b) and atom locations $(c, d)$, squares : Au spots $(a, b)$ and atom locations $(c, d)$.
2.2. STRUCTURE: ATOMIC DIAMETER INFLUENCE. - Turning to the influence of atomic size on the structure of the adsorbate, it is obvious that a pseudomorphic layer can be formed only if the atomic diameter $d_{\mathrm{a}}$ of the adsorbate is comparable or smaller than the atomic diameter of the substrate $d_{s}$. $\mathrm{Pd}$ with $d_{\mathrm{a}}=2.75 \AA$ and $\mathrm{Cu}$ with $d_{\mathrm{a}}=2.56 \AA$ vs $d_{\mathrm{s}}=2.74 \AA$ are good examples for such adsorbates. If $d_{\mathrm{a}}$ is much smaller than $d_{\mathrm{s}}$, then a $(1 \times 1)$ structure may but must not occur at a coverage when $N_{\mathrm{a}} \approx N_{\mathrm{s}}$. An example is $\mathrm{Be}\left(d_{\mathrm{a}}=2.29 \AA\right)$ which shows a $p(9 \times 1)$ LEED pattern (Fig. $3 a)$ up to $N_{\mathrm{a}} \approx 11 \times 10^{14} \mathrm{~cm}^{-2}$, a $c(2 \times 9)$ pattern (Fig. $\left.3 b\right)$ at high coverages and a diffuse $(1 \times 1)$ pattern in between at $N_{\mathrm{a}} \approx N_{\mathrm{s}}[17]$. These patterns can be attributed to multiple scattering between the substrate and surface layers with the unit meshes shown in figures $3 c$ and $3 d$. Both structures are distorted hexagonal nets with

$$
N_{\mathrm{a}}=\frac{7}{9} \sqrt{2} \frac{1}{a_{\mathrm{w}^{2}}}=11.0 \times 10^{14} \mathrm{~cm}^{-2}
$$

and

$$
N_{\mathrm{a}}=\frac{14}{9} \sqrt{2} \frac{1}{x_{\mathrm{w}^{2}}}=22.0 \times 10^{14} \mathrm{~cm}^{-2} \approx N_{\mathrm{Be}}(0001)
$$

respectively. Two conclusions can be drawn from these observations : 1) the surface adsorbs as much $\mathrm{Be}$ as the diameter (2.29 $\mathrm{A})$ of the Be atoms allows, a)
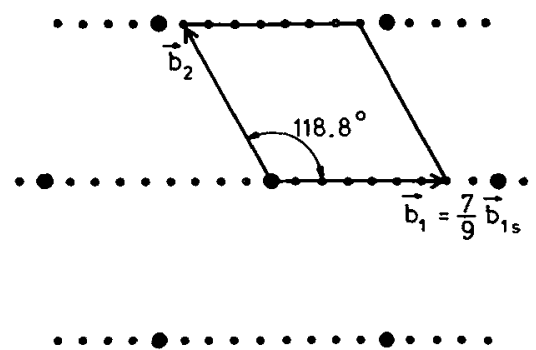

[001]

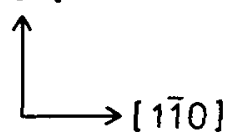

무

[110]

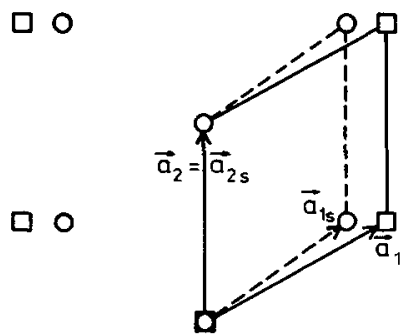

c)
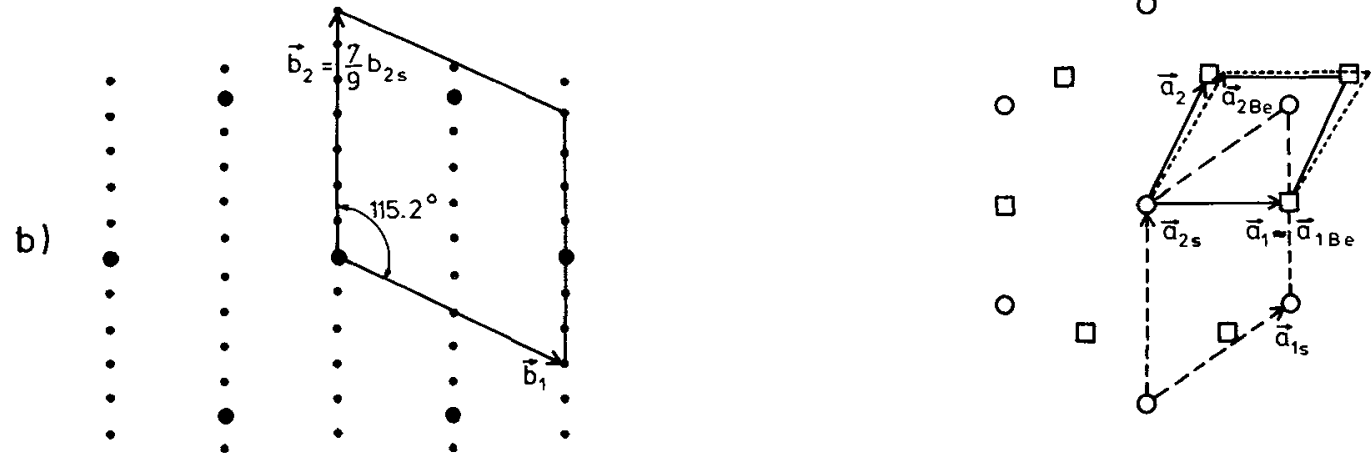

FIG. 3. - LEED patterns (a,b) and unit meshes (c, d) of Be on W(110) $\left.a, c) N \leqslant 11 \times 10^{14} \mathrm{~cm}^{-2}, c, d\right) N \rightarrow 22 \times 10^{14} \mathrm{~cm}^{-2}$. In $d$ ) the unit mesh of the $\mathrm{Be}(0001)$ plane is shown too (dotted lines). Solid lines : Be, dahed lines : W: 
2) no island formation of close-packed twodimensional $\mathrm{Be}$.islands occurs at low coverage but rather a hexagonal lattice with a much larger interatomic distance $d_{\mathrm{a}} \approx 3.17 \AA$. Such hexagonal lattices with large interatomic spacings, which decrease continously with coverage, are typical for large adsorbed atoms with large dipole moments such as $\mathrm{Cs}$ or $\mathrm{Ba}$ (see above). In these adsorbates the order is attributed to the repulsive forces between the dipoles, influenced more (in the case of $\mathrm{Ba}$ ) or less (in the case of $\mathrm{Cs}$ ) by the periodic forces exerted by the substrate. This suggests that the low coverage structure of Be (Fig. 3a,c) is also caused in part by repulsive forces. This suggestion is supported by single plane field emission work function measurements [31] which show a strong $\phi$ decrease with $\mathrm{Be}$ coverage followed by a minimum. Still another dependence of film structure on coverage has been observed in $\mathrm{Pb}$ adsorbates [2] : a continous deformation and rotation of the unit mesh (Fig. 4). Throughout this process the $\mathrm{Pb}$ atoms are lined up in rows parallel to the most densily packed substrate directions $(\{\overline{1} 11\})$. The initial phase of this process has been overlooked in earlier work [18]. Similarily a density-independent hexagonal packing has been reported in the first study of Y on W(110) [14] while a more detailed examination [25] revealed a compression increasing with coverage. Thus considerable caution is in place in the analysis of the reported structures.

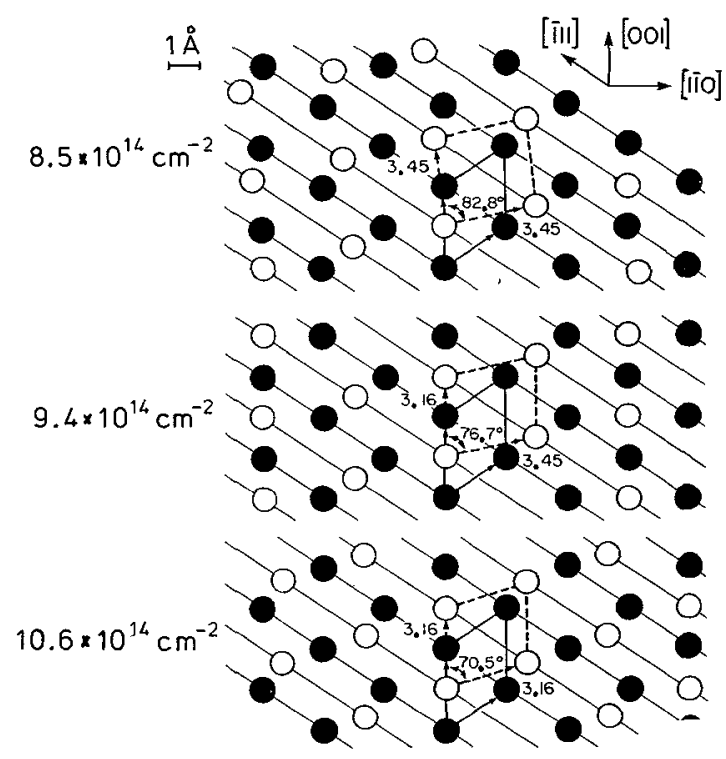

FIG. 4. - Rotation and deformation of $\mathrm{Pb}$ adsorbate unit mesh on $\mathrm{W}(110)$ with increasing atomic density $N$. Open circles $\mathrm{Pb}$, full circles $\mathrm{W}$. The lateral position of the $\mathrm{Pb}$ layer with respect to the

W surface cannot be deduced from the LEED geometry.

2.3. Surface POTENTIAL: LARGE $\Delta X$. - The reasons for the structural changes have to be sought in the lateral interactions and their competition with the adsorbate-substrate interactions. The forces range from purely ionic to predominantly covalent. Ionic forces are predominant in adsorbates with large $\Delta X$-values at low and intermediate coverages, covalent forces at high coverages and in adsorbates with small $\Delta X$ values. A good indicator for the type of bonding is the dipole moment per adsorbed atom. Form $\left(\frac{\mathrm{d} \phi}{\mathrm{d} N}\right)_{N \rightarrow 0}$ the following zero coverage limit dipole moments can be deduced : $19 \mathrm{D}$ [26], $14 \mathrm{D} \mathrm{[6]}$ or $10 \mathrm{D}[27]$ for $\mathrm{Cs}, 9.5 \mathrm{D}[24]$ or $13.5 \mathrm{D}$ [8] for $\mathrm{Ba}$. If the dipole is attributed to a point charge $q . e$ in the distance $r$ of the effective surface and its image charge, then $\mu=9.6 q . r$. Assuming $q=1$ leads to $r=2.03,1.46$ or 1.04 for $\mathrm{Cs}$ and $r=0.99$ or $1.41 \AA$ for $\mathrm{Ba}$. These values have to be compared with ionic radii $r_{\mathrm{Cs}}^{+}=1.69 \AA$ and $r_{\mathrm{Ba}}^{+}\left(1.14 \AA=r_{\mathrm{Ba}}^{++}<r_{\mathrm{Ba}}^{+}<r_{\mathrm{Ba}}=2.18 \AA\right) \quad$ which shows that at least for $\mathrm{Cs}$ the larger $r$-values derived from $\mu$ are quite compatible with full ionization. The dipole moments at the work function minimum $\mu=6.13 \mathrm{D}[6]$ or $5.33 \mathrm{D}[27]$ for $\mathrm{Cs}$ and 7.3 D [24] or $8.1 \mathrm{D}[8]$ for $\mathrm{Ba}$ suggest that there is still considerable charge on the adsorbate atoms. It is, however, more appropriate to compare the minimum work functions $\phi_{\mathrm{Cs}}^{\min }=1.60 \mathrm{eV}$ and $\phi_{\mathrm{Ba}}^{\min }=1.70 \mathrm{eV}$ with the work functions of the bulk adsorbate $\phi_{\mathrm{Cs}}^{\mathrm{B}}=1.81 \mathrm{eV}$ and $\phi_{\mathrm{Ba}}^{\mathrm{B}}=2.49 \mathrm{eV}$ or with the monolayer work functions $\phi_{\mathrm{cs}}^{\mathrm{sat}}=2.1 \mathrm{eV}$ and $\phi_{\mathrm{Ba}}^{\mathrm{sat}}=2.63 \mathrm{eV}$ instead of comparing it with that of the clean W surface $(5.3 \mathrm{eV})$. Then it is seen that the surface dipole is not much larger than that of the bulk adsorbate. Therefore, little charge transfer to the substrate has to be involked at and beyond the work function minimum. Rather, the Cs electrons are increasingly concentrated in the adsorption layer. This is supported by energy loss experiments which show that above $\phi_{\mathrm{Ba}}^{\min } \mathrm{Ba}$ plasmons are excited [28]. The probability of plasmon excitation increases proportional to the coverage up to 1 monolayer. In the case of $\mathrm{Cs}$, plasmon creation commences already at the coverage at which neutrals begin to be observed in thermal desorption, i.e. at $N \approx 0.7 \times 10^{14} \mathrm{~cm}^{-2}$, well below the coverage $N^{\text {min }}=3.3 \times 10^{14} \mathrm{~cm}^{-2}$. These changes of the electron distribution with coverage cause corresponding changes of the heat of adsorption/atom $E_{\mathrm{a}}$ with coverage. Figure 5 illustrates this for $\mathrm{Ba}$ with data derived from references $[8,9,10$ and 28$]$. The rapid decrease of $E_{\mathrm{a}}$ above $N^{\mathrm{min}}$ clearly is due to the metallization of the layer indicated by the increasing plasmon loss probability $\left(A_{\mathrm{p}}\right)$. In the case of Cs on W(110) a much less pronounced shoulder in $E_{\mathrm{a}}=E_{\mathrm{a}}(N)$ has been reported for $N$ considerably below $N^{\text {min }}$ [6]. This correlates well with the earlier onset of metallization indicated by the appearance of the plasmon peak in the energy loss spectrum. Thus the variation of the heat of adsorption with atomic density can be understood on the basis of the decrease of the 
charge per adsorbed atom. There is however still enough repulsion between these charges - or charge-image charge dipoles - above $N^{\text {min }}$ to assure a hexagonal lattice.

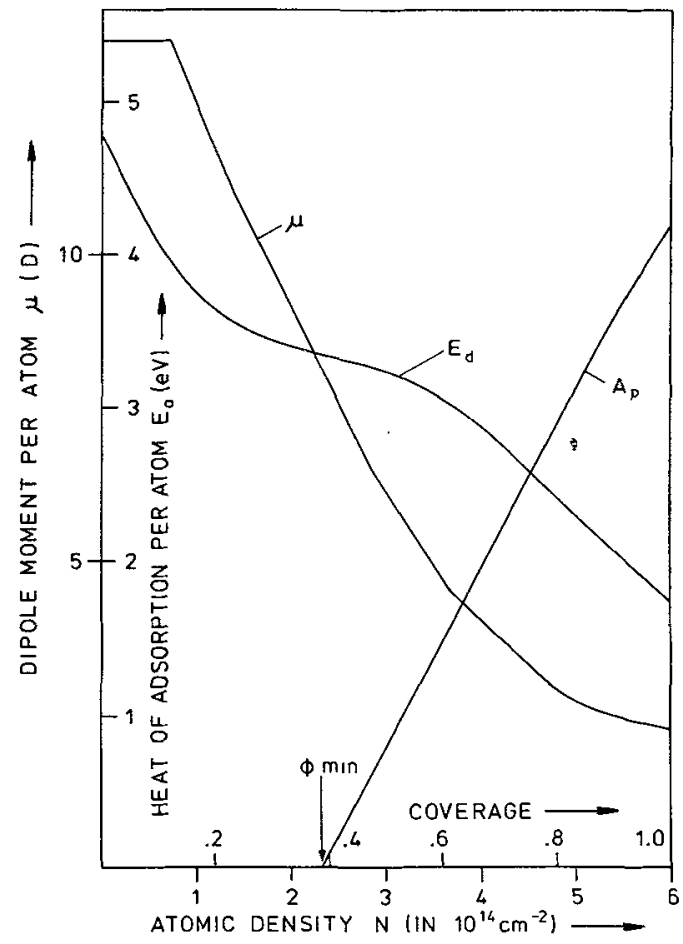

FrG. 5. - Dipole moment $\mu(\mathrm{D})$, heat of adsorption $E_{\mathrm{a}}(\mathrm{eV})$ and $3.5 \mathrm{eV}$-plasmon loss intensity $\left(A_{\mathrm{p}}\right)$ of $\mathrm{Ba}$ on $\mathrm{W}(110)$ as a function of atomic density $N$

2.4. Surface potential: SMall $\Delta X$. - In adsorbates with small $\Delta X$ the situation is more complex. Here little charge transfer is expected but dipole formation due to the Smoluchowski effect (S.E.). This is illustrated by the fact that adsorbed $\mathrm{W}$ atoms $(\Delta X=0)$ have a dipole moment of $0.7 \mathrm{D}$ [33], $0.3 \mathrm{D} \mathrm{[34]}$ or $0.2 \mathrm{D}$ [35]. On the other hand, for Ir and $\operatorname{Pt}\left(X_{\mathrm{Ir}}, X_{\mathrm{pt}}>X_{\mathrm{w}}\right) \mu=0$ has been measured [35]. Here clearly the electronegativity $X$ is not a very useful quantity. Instead a more sophisticated quantum mechanical treatment is called for. Using Gadzuk's formula, $\mu$ values of $0.23 \mathrm{D}, 0.16 \mathrm{D}$ and $0.16 \mathrm{D}$ have been estimated for $\mathrm{W}$, Ir and $\mathrm{Pt}$ respectively [35] instead of the zero or negative $\mu$ values expected from electronegatively considerations. Negative initial $\mu$ values on an atomically smooth surface appear also unlikely on the basis of the Smoluchowski effect which produces a positive dipole in an adsorbed atom. Thus the work function should decrease initially, irrespective of whether $X_{\mathrm{a}} \gtreqless X_{\mathrm{s}}$. This is confirmed by experiment $[2,5,22-24,34]$ (see Fig. 6). In all adsorbates with $X_{\mathrm{a}}<X_{\mathrm{s}} \phi$ decreases initially approximately linearily with $N$ but for $N \rightarrow N^{\text {sat }}$ more or less pronounced deviations towards higher $\phi$ from linearity occur increasing from $\mathrm{Cu}[22]$ to

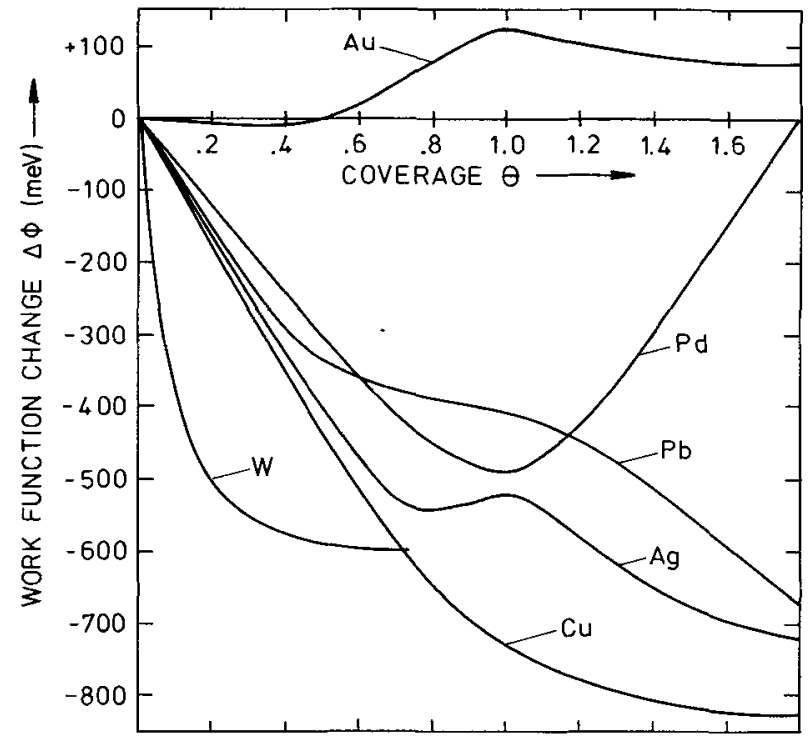

FIG. 6. - Change of the work function of a W(110) surface due to adsorption of $\mathrm{Ag}, \mathrm{Au}, \mathrm{Cu}, \mathrm{Pb}, \mathrm{Pd}$ and $\mathrm{W}$ at $300 \mathrm{~K}$.

$\operatorname{Ag}[5,32]$ (which shows a small local $\phi$-maximum at $N^{\text {sat }}$ ) to $\mathrm{Pb}$ [2]. The curves may be considered to be due to the superposition of two phenomena : 1) a general decrease of $\phi$ caused by electron transfer due to the electronegativy difference and 2) an atomic roughness-dependent contribution caused by the Smoluchowski effect. Because of the dominance of the first contribution a reliable distinction between random adsorption and island formation is not possible. In the case of $\mathrm{W}$ adsorption $\left(X_{\mathrm{a}}=X_{\mathrm{s}}\right)$, however, a transition from predominant random adsorption at low temperature to island growth at higher temperature can be deduced from $\phi$ ( $N$ ) [34]. In Au [34] adsorption $\left(X_{\mathrm{a}}-X_{\mathrm{s}}\right)$ the influence of $\Delta X$ and of the S.E. counteract each other. An exception is Pd, for which also $X_{\mathrm{a}}>X_{\mathrm{s}}$ but for which $\phi$ decreases up to one monolayer. The initial decrease which gives a dipole moment/atom of $\mu=0.2 \mathrm{D}$ can be rationalized using Gadzuk's $\mu$ formula or the S.E. but the positive $\mu$ at one monolayer is unexpected even on the basis of the most recent quantum mechanical calculations [36].

2.5. ENERGETICS : SMALL $\Delta X$. - In all cases the work function changes are sufficiently small so that covalent bonding can be assumed. This justifies attempts to determine the structure of the adsorbate theoretically by minimizing the total energy of the system consisting of the atoms in the adsorbate and in the topmost substrate layers assuming pairwise interaction potentials of the Lennard-Jones, Mie or Morse type. Calculations assuming a rigid substrate [37, 38] or allowing relaxation of the topmost layer $[39,40]$ have been made for $\mathrm{Pb}$ and $\mathrm{Cu}$ on $\mathrm{W}(110)$. For a $\mathrm{Cu}$ monolayer, substrate periodicity is obtained with relaxation [40], a structure with match in one direction and mismatch in the other on the rigid substrate [37]. For the $\mathrm{Pb}$ 
layer with $N=9.4 \times 10^{14} \mathrm{~cm}^{-2}-$ which has a $(3 \times 1)$ structure - the two calculations give two different atom distributions in the superstructure unit mesh [38, 39] (Fig. 7). The calculated adsorption energies, e.g. $3.95 \mathrm{eV}$ and $4.99 \mathrm{eV}$ for $\mathrm{Cu}$ for $N \rightarrow 0$ and $N^{\text {sat }}=N_{\mathrm{s}}[40], 2.31 \mathrm{eV}$ and $2.65 \mathrm{eV}$ for $\mathrm{Pb}$ for $N \rightarrow 0$ and $N=9.4 \times 10^{14} \mathrm{~cm}^{-2}$ agree semiquantitatively with the values deduced from thermal desorption. Particularily encouraging results are obtained with Morse potentials and allowance for relaxation of the topmost substrate layer. Thus it appears that such calculations may allow meaningful predictions. One of these predictions is the island growth of $\mathrm{Cu}$ on W(110).

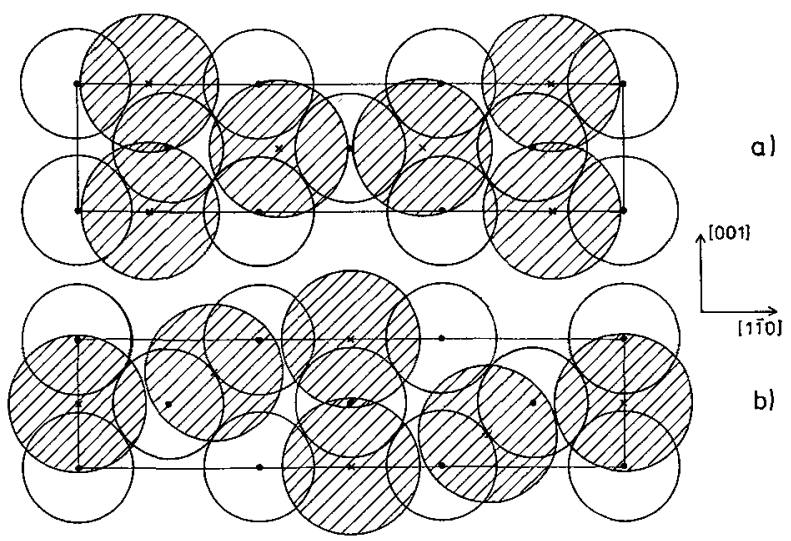

FIG. 7. - Rectangular superstructure unit meshes of the structures proposed for the $\mathrm{p}(3 \times 1)$ layer of $\mathrm{Pb}$ on $\mathrm{W}(110)$. Open circles : $\mathrm{W}$ atoms, hatched circles : $\mathrm{Pb}$ atoms.

There is also experimental evidence for significant stability of islands. Analysis of the thermal desorption spectra of $\mathrm{Cu}$ on W(110) $[22,41]$ shows a break in the $\ln \tau v s 1 / T$ curves at approximately $T_{\mathrm{c}}=1200 \mathrm{~K}$ with $T_{\mathrm{c}}$ slightly increasing with $N$ (Fig. 7). The evalution of the data above $T_{\mathrm{c}}$ give desorption energies $E_{\mathrm{a}}$ increasing linearly from $3.38 \mathrm{eV}$ at $N \rightarrow 0$ to $4.12 \mathrm{eV}$ at $0.6 \mathrm{~m} . \mathrm{l} . \triangleq N=8.3 \times=10^{14} \mathrm{~cm}^{-2}$ and frequency factors $\nu$ increasing from $1 \times 10^{13} \mathrm{~s}^{-1}$ to $2.5 \times 10^{15} \mathrm{~s}^{-1}$ in the same $N$-range, assuming first order desorption. This can be understood in terms of desorption from a two-dimensional gas of atoms with attractive interactions $E_{2}$. With increasing density $E_{\mathrm{d}}$ increases because of the increasing $E_{2}$ contributions, and $\nu$ increases because of the continous transition from mobile to immobile adsorption. Below $T_{\mathrm{c}}$ the evaluation indicates that $E_{\mathrm{d}}$ rises from an extrapolated value of $3.5 \mathrm{eV}$ for $N \rightarrow 0$ to a value of approximately $4.25 \mathrm{eV}$ which is constant from approximately $0.15 \mathrm{~m}$.1. to $0.6 \mathrm{~m} .1$. $\nu$ also increases initially to a value of approximately $1 \times 10^{16} \mathrm{~s}^{-1}$ but decreases slightly in the range in which $E_{\mathrm{d}}$ is constant assuming first order adsorption. This can be attributed to desorption from an immobile two-dimensional $\mathrm{Cu}$ crystal. The energy per lateral bond is estimated from these data

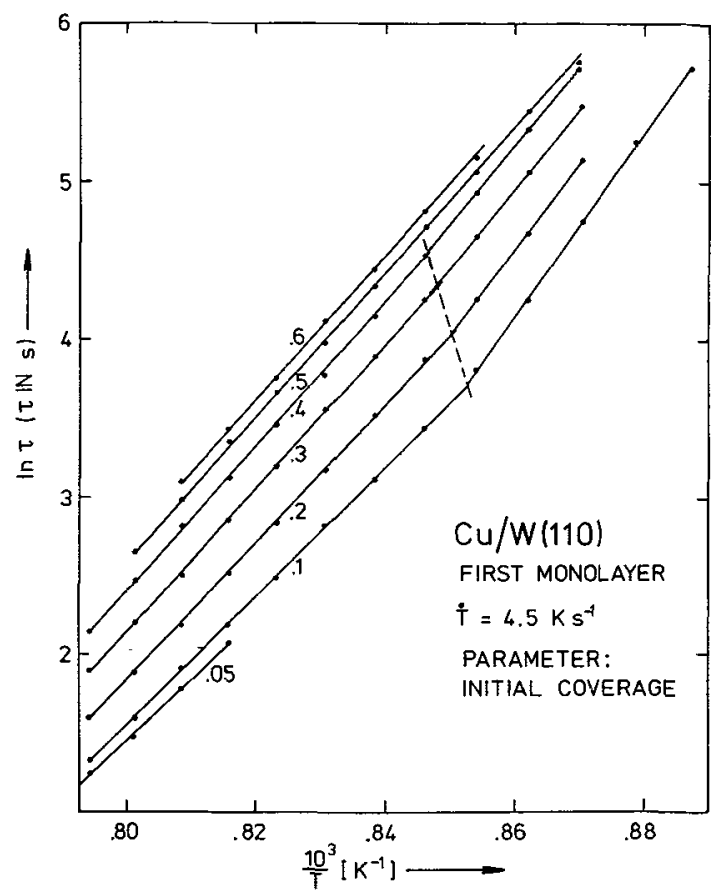

FIG. 8. - Mean lifetime vs reciprocal temperature for $\mathrm{Cu}$ on $\mathrm{W}(110)$.

at $E_{2} \approx 0.15 \mathrm{eV}$. A comparison of the less detailed $\mathrm{Ag}$ and $\mathrm{Au}$ desorption data with those of $\mathrm{Cu}$ suggests that also in these adsorbates desorption occurs at least in part from islands. If the frequently used assumption is made that $\nu=1 \times 10^{13} \mathrm{~s}^{-1}$ independent of $N$, then $E_{\mathrm{d}}=3.15 \mathrm{eV}$ for $\mathrm{Ag}$ and $3.43 \mathrm{eV}$ for $\mathrm{Cu}$ in good agreement with the values $E_{\mathrm{d}}=3.0 \mathrm{eV}$ and $3.4 \mathrm{eV}$ obtained from adsorption isobars [24]. This assumption however neglects the change of the configuration of the desorbing atoms and its potential well with increasing $N$. (In the case of alkali and alkaline earth adsorbates the assumption $\nu=1 \times 10^{13} \mathrm{~s}^{-1}$ independent of coverage is also frequently made in evaluating a single adsorption isobar to arrive at $E_{\mathrm{d}}$. However measurements made for several incident fluxes indicate that this assumption is apparently justified for these adsorbates within the limits of error. It is not understood at present why this is so).

2.6. OTHER ADSORBATE MODELS. - There are other theoretical models discussing the structure of the adsorbate as a function of coverage or the structure of the complete monolayer. One [42] of them is limited to adsorption with significant charge transfer (group I adsorbates) and allows only general statements on phase transitions in these films. The indirect interaction model [43] which qualitativeley predicts a certain sequence of ordered structures for (100) surfaces is not very appropriate for the smooth (110) surfaces. Definite structure proposals are possible with Hubers model [46-48] of superstructures which is based on two principles : 1) high symmetry of the coincidence mesh and 
2) minimum number of different kinds of adsorption sites (this emphasizes the importance of the local bonding configuration !). This model seems to be quite useful in the case of surfaces which have a considerable potential variation (e.g. the (100) surface), in particular if adsorbate and substrate form compounds in the bulk. It appears to be less applicable to the (110) surface with its rather smooth potential and the repulsive (group I limit) or attractive (group II limit) forces between atoms adsorbed on this surface. An example is shown in figure 7. In structure $a$ ) all $\mathrm{Pb}$ atoms are adsorbed in the same kind of adsorption sites (Huber model), in structure $b$ ) in two different sites $B$ and $C$. According to older pairwise interaction calculations $[44,45]$ there is little difference in binding energy between sites $A$ and $B$ but in site $C$ saddle position it is about $10 \%$ lower than in site $\mathrm{A}$. Therefore structure $a$ ) seems to be favored. In favor of structure $a$ ) is also the smaller compression necessary to accomodate the $\mathrm{Pb}$ atoms in structure $b$ ). The $\mathrm{Pb}-\mathrm{Pb}$ distance along the [001] direction is the same in both structures $(3.16 \AA$ instead of $3.5 \AA$ in bulk $\mathrm{Pb}$ ) but the other $\mathrm{Pb}-\mathrm{Pb}$ distance is only $3.45 \AA$ in structure $b$ ) which is $1.4 \%$ smaller than the diameter $d_{\mathrm{Pb}}$ in metallic $\mathrm{Pb}$. In structure $a$ ) the other $\mathrm{Pb}-\mathrm{Pb}$ distances are $3.542 \AA$ and $3.538 \AA$ which is approximately $1.1 \%$ larger than $d_{\mathrm{Pb}}$. A distinction between the two structures must await more detailed calculations, for LEED can at present not distinguish between them due to the strong double scattering evident in the diffraction patterns. Finally, the most recent model, the static distortion wave (SDW) model [49], which is an analogon to the charge density waves (SDW) in solids, is very attractive but not developed far enough to allow predictions for the kind of systems discussed in this paper. Therefore one is left with experiments, if general rules are to be formulated.

3. General empirical rules for structure, surface potential and energetics. - 3.1. STRUCTURE RULES. - Such rules can use the type of bonding as a classification parameter or the atomic diameter. The type of bonding may be characterized by the ratio (or the difference) of $\Delta X^{2}$ and $\frac{1}{12}\left(E_{\mathrm{sa}}+E_{\mathrm{ss}}\right)=E_{\mathrm{a}}^{\prime}$, with the limiting cases $\Delta X^{2} \gg E_{\mathrm{a}}^{\prime}$ and $\Delta X^{2} \ll E_{\mathrm{a}}^{\prime}$ (see Table I). Consider first the lateral interactions. When $\Delta X<E_{\mathrm{a}}^{\prime}$, in particular for $\Delta X^{2}>E_{\mathrm{a}}^{\prime}$, considerable charge transfer between adsorbate and substrate occurs so that dipoles are formed with mutual repulsion. This results in a hexagonal lattice whose lattice constant is continously decreasing with increasing coverage. When $\Delta X^{2} \ll E_{\mathrm{a}}^{\prime}$ the attractive forces between the adsorbate atoms lead to formation of two-dimensional islands with a structure which minimizes the strain energy. This may be a pseudomorphous layer (when $d_{\mathrm{a}} \leqslant d_{\mathrm{s}}$ ) or a distorted close packing with a distortion such that the distance between neighbouring rows of atoms parallel to $\langle 1 \overline{1} 1\rangle_{\mathrm{s}}$ is the same in adsorbate and substrate. A transition from pseudomorphic structure to this distorted close packing may occur with increasing coverage (compression!) or temperature, if the mobility at lower temperatures is insufficient to form this packing. Island formation may not occur at very low temperature due to limited surface diffusion. Thus surface diffusion of $\mathrm{Pd}$ atoms $\left(E_{\mathrm{d}}=\mathbf{0 . 4 9} \pm 0.04 \mathrm{eV}\right.$, $\left.D_{0}=7 \times 10^{-4} \mathrm{~cm}^{2} \mathrm{~s}^{-1}\right)[48]$ is very slow below $160 \mathrm{~K}$ and of $\mathrm{W}$ atoms $\left(E_{\mathrm{d}}=0.91 \pm 0.05 \mathrm{eV}\right.$, $D_{\mathrm{o}}=2 \times 10^{-3} \mathrm{~cm}^{2} \mathrm{~s}^{-1}$ ) [49] below $300 \mathrm{~K}$. At very high temperature, islands may become unstable before significant desorption starts.

The interaction with the periodic substrate competes with the lateral interactions and tries to impress its periodicity or multiples of it onto the adsorbate. When $\Delta X^{2} \notin E_{\mathrm{a}}^{\prime}$ the substrate influence a) determines the azimuthal orientation of the hexagonal lattice, $b$ ) deforms this lattice with increasing coverage by imposing on it a fixed interatomic distance in one direction or $c$ ) causes the formation of several deformed hexagonal lattices with fixed dimensions independent of coverage. Thus a series of primitive or centered $(m \times n)$ structures is formed two of which exist simultaneously at coverages between those corresponding to two successive completely filled structures. The substrate influence in the case $\Delta X^{2} \ll E_{a}^{\prime}$ has already been mentioned above. In addition, due to the strong interaction with the substrate, one or more stable or metastable adsorption layers may form on top of the first layer. This process may leave the structure of the first layer untouched to a first approximation (e.g. in $\mathrm{Cu}$ ) or may induce a new structure (e.g. in $\mathrm{Ag}, \mathrm{Au}$ ).

The atomic diameter is an important factor only at high coverages : it determines the maximum atomic density $N_{\mathrm{a}}^{\text {sat }}$. If the hexagonal close packing of the adsorbate atoms has a smaller packing density $N_{\mathrm{a}}^{\text {hcp }}$ than the substrate surface $\left(N_{\mathrm{s}}\right)$, then the saturation layer is generally slightly compressed $\left(N_{\mathrm{a}}^{\mathrm{sat}}>N_{\mathrm{a}}^{\mathrm{hcp}}\right)$. If $N_{\mathrm{a}}^{\text {hcp }} \gtrsim N_{\mathrm{s}}$ then usually $N_{\mathrm{a}}^{\text {sat }} \leqslant N_{\mathrm{s}}$.

\subsection{RULES GOVERNING SURFACE POTENTIAL} AND ENERGETICS. - For the surface potential the parameters electronegativity difference $\Delta X$ and atomic roughness are appropriate. If $\Delta X \nless 0$ charge transfer to or from the substrate will occur which leads to dipole formation and a workfunction increase $\left(X_{\mathrm{a}}>X_{\mathrm{s}}\right)$ or decrease $\left(X_{\mathrm{a}}<X_{\mathrm{s}}\right)$. The dipole moment $\mu$ decreases with increasing atomic density $N$ due to depolarization. $\mu$ is essentially only a function of $N$ and does not depend on the long range order. For large $\Delta X=X_{\mathrm{s}}-X_{\mathrm{a}} \geqslant 1.8$ the surface potential passes through a minimum, for $\Delta X \lesssim 1.8$ it decreases monotonically. The saturation 
coverage dipole moment is only approximately determined by $\Delta X$. The transition from largely ionic to predominantly covalent bonding begins at the atomic density at which plasmon excitation commences.

Superimposed on this $\Delta X$-determined surface potential change is the atomic roughness-determined surface potential change. If $\Delta X$ is large it gives only a small contribution but if $\Delta X=0$ it is the only contribution within this highly simplified description. In all cases it causes a dipole with the positive side outward and is strongest if atoms are adsorbed at random (linear coverage dependence at low coverage). If islands are formed only the edge atoms contribute so that the surface potential changes like the square root of the coverage until the islands merge. In both cases the surface potential change becomes approximately zero at the completion of a monolayer. In multilayer adsorption this causes an oscillatory structure superimposed on the $\Delta X$-determined overall change.

If $\Delta X^{2} \ll E_{\text {a }}^{\prime}$, then the dipole-dipole repulsion increases with coverage, the image force attraction decreases. As a consequence the heat of adsorption $E_{\text {a }}$ decreases rapidly with coverage. The decrease slows down near the coverage at which the transition from ionic to covalent bonding begins. Beyond this coverage $E_{\mathrm{a}}$ approaches rapidly values close to the sublimation energy of the bulk of the adsorbate. If the lateral interactions are attractive as is usually the case when $\Delta X^{2} \ll E_{\text {a }}^{\prime}$, then $E_{\text {a }}$ increases rapidly with coverage due to the decreasing distance between atoms. A constant value is reached upon island formation. Multiple binding states can be formed if multilayer adsorption occurs which is the rule for high $E_{\mathrm{a}}$

4. Summary. - The discussion of the experimental results in section 2 has shown a wide variety of phenomena and a lack of data for sufficient characterisation of the adsorbates as to allow the derivation of quantitative general empirical rules. Consequently only qualitative rules have been presented in section 3 . These rules have obvious exceptions. Thus adsorption of $\operatorname{Be}\left(X_{\mathrm{a}}=1.5\right)$ produces a workfunction minimum while $\mathrm{Mg}$ adsorption $\left(X_{\mathrm{a}}=1.2\right)$ causes only a monotonic $\phi$ decrease. A monolayer of $\operatorname{Pd}\left(X_{\mathrm{a}}=2.2\right)$ lowers $\phi$ by approximately $0.5 \mathrm{eV}$ although $. X_{\mathrm{a}}<X_{\mathrm{s}}=2.0$ and $\phi_{\mathrm{Pd}(111)}=5.4 \mathrm{eV}>\phi_{\mathrm{w}(110)}=5.3 \mathrm{eV}$. It should also be noted that $X_{\mathrm{s}}=2.0$ has to be assumed for the $W(110)$ surface instead of the free $W$ atom value of $X_{\mathrm{w}}=1.7$. These examples show that predictions based on the standard $X$ values, as useful they are in general, can be wrong in specific cases.

Except for these cases the qualitative rules describe well the general behaviour of metal adsorbates on $\mathrm{W}\{110\}$ surfaces. Generalizations to atomically rougher surfaces such as $\{100\}$ or $\{211\}$ surfaces or to surfaces with low surface energy have to be done with caution, however.
[1] Yonehara, K. and SCHMIDT, L. D., Surf. Sci. 25 (1971) 238.

[2] Bauer, E., Poppa, H. and Todd, G., Thin Solid Films 28 (1975) 19

[3] Felter, T. E., Barker, R. A. and Estrup, P. J., Phys. Rev. Lett. 38 (1977) 1138.

[4] Cetronio, A. and Jones, J. P., Surf. Sci. 44 (1974) 109.

[5] Bauer, E., Poppa, H., Todd, G. and Davis, P. R., J. Appl. Phys., submitted.

[6] Pedorus, A. G. and Naumovets, A. G., Sov. Phys.-Solid State 12 (1970) 301.

[8] GoRODETSKII, D. A. and MEL'NIK, Yu. P., Surf. Sci. 62 (1977) 647.

[9] Gorodetskit, D. A. and Mel'Nik, Yu. P., Sov. Phys.-Solid State 16 (1975) 1805.

[10] Fedorus, A. G., Naumovets, A. G. and Vedula, Yu. S., Phys. Status Solidi 13 (1972) 445.

[11] GorodetskiI, D. A., Mel'NIK, Yu. P. and Yas'Ko, A. A., Ukr. Fiz. Zh. 12 (1967) 646.

[12] Medvedev, V. K. and Smereka, T. P., Sov. Phys.-Solid State 16 (1974) 1046.

[13] GorodetskiI, D. A., Yas'ko, A. A. and Shevlyakoy, S. A., Izv. Akad. Nauk. SSSR, Ser. Fiz. 35 (1971) 436.

[15] Gorodetskir, D. A. and FUNG-Kho, Ukr. Fiz. Zh. 14 (1969) 94.

[16] GoRoDETSKI, D. A. and YAS'Ko, A. A., Sov. Phys.-Solid State 10 (1969) 1812.

[17] NiehUS, H., PhD thesis, Clausthal (1975).

[18] Gorodetskil, D. A. and Yas'Ko, A. A., Sov. Phys.-Solid State 11 (1969) 640.
[19] Gorodetskil, D. A. and Yas'Ko, A. A., Sov. Phys.-Solid State 13 (1971) 1085

[20] BoIko, B. A., GorodeTSKIl, D. A. and Yas'Ko, A. A., Sov. Phys.-Solid State 15 (1974) 2101.

[21] Bauer, E. and Poppa, H., Thin Solid Films 12 (1972) 167.

[22] Bauer, E., Poppa, H., Todd, G. and BonCzeK, F., J. Appl. Phys. 45 (1974) 5164.

[23] Paraschkevov, D., Schlenk, W., Bajpai, R. P. and Bauer, E., Proc. 7th Intern. Vac. Congr. and 3rd Internat. Conf. Solid Surfaces, Vienna 1977.

[24] Vedula, Yu. S. and Naumovets, A. G., Ukr. Fiz. Zh. 43 (1973) 1000.

[25] Voronin, V. B. and Naumovets, A. G., Izv. Akad. Nauk SSSR, Ser. Fiz. 35 (1971) 325.

[26] Lee, T. E. and Stickney, R. E., Surf. Sci. 32 (1972) 100.

[27] Desplat, J.-L., Ann. Rep., NASA Contr. NAS 2-9001, Oct. 1976.

[28] GoRodetSkiI, D. A., GorChinskit, A. D., MAKSIMENKo, V. I. and MEL'NIK, Yu. P., Sov. Phys.-Solid State 18 (1976) 691.

[29] Kanash, O. V., Naumovers, A. G. and Fedorus, A. G. Sov. Phys. -JETP 40 (1975) 903.

[30] Naumovets, A. G. and Fedorus, A. G., Sov. Phys.-JETP 41 (1976) 587

[31] Polanski, J., Sidorski, Z. and Zuber, S., Acta Phys. Pol. A49 (1976) 299.

[32] KolaczkIEWICZ, J. and SidoRki, Z., Surf. Sci. 63 (1977) 501 .

[33] Plummer, E. W. and Rhodin, T. N., Appl. Phys. Lett. 11 (1967) 194 
[34] Besocke, K. and Wagner, H., Phys, Rev. B 8 (1973) 4597

[35] Kellogg, G. L. and Tsong, T, T., Surf. Sci. 62 (1977) 343.

[36] Leynaud, M. and Allan, G., Surf. Sci. 53 (1975) 359.

[37] Halıcioğlu, T., Thin Solid Films 32 (1976) 169.

[38] HalicioĞLU, T, private comm.

[39] Stoop, L. C. A., Thin Solid Films 42 (1977) 33.

[40] Stoop, L. C. A., Proc. 7th Intern. Vac. Congr. and 3rd Internat. Conf. Solid Surfaces, Vienna 1977.

[41] Bauer, E., Bonczek, F., Poppa, H. and Todd, G., Surf. Sci. 53 (1975) 87

[42] Bol'shov, L. A. and Napartovich, A. P., Sov, Phys.-JETP 37 (1973) 713.
[43] Huber, M. and Oudar, J., Surf. Sci. 47 (1975) 605

[44] Biberian, J. P. and Huber M., Surf. Sci. 55 (1976) 259.

[45] Biberian, J. P., Surf. Sci. 59 (1976) 307.

[46] Einstein, T. L. and Schrieffer, J. R., Phys. Rev. B 7 - (1973) 3629

[47] Novaco, A. D. and MCTaque, J. P., Phys. Rev. Lett. 38 (1977) 1286.

[48] BENECKE, W., M. S. thesis, Clausthal 1977.

[49] Bassett, D. W. and Parsley, M. J., J. Phys. D 3 (1970) 707

\section{DISCUSSION}

J. OUDAR. - How many layers are necessary to obtain the work function of the bulk of the deposit material ?

E. BAUER. - This depends upon the deposit material and the experimental conditions which determine the atomic roughness of the layer. Usually, 3-5 layers are required.

S. C. YING. - I don't quite agree with the criterion that when the bond is mainly covalent one can use the pairwise interaction approximation. In the last two or three years, first principle calculations of $H$ chemisorbed on simple metals and semiconductors have indicated the basic many-body nature of the adsorbate-substrate interaction even though the bonding is covalent in nature.

E. BAUER. - I said that the pairwise interaction approximation is useful only when there is little charge transfer. Its usefulness is demonstrated by recent calculations by Halicioğlu [37] and Stoop [39]. Many-body effects are implicitly included in the usual Lennard-Jones, Morse or Mie potentials. I agree that explicit consideration of many-body interactions is necessary for a more basic understanding of adsorption even when there is little charge transfer. Thus the work function change cannot be obtained from pairwise interaction potentials. Until first principle calculations become available, these potentials remain useful.

J. G. DASH. - You have described two systems in which bulk phase formation occurs earlier at higher temperatures. Have you also seen systems in which the reverse occurs?

E. BAUER. - No.
B. MUTAFTSCHIEV. - You mentioned that in the case of gold deposition on $W$, the mass time curves show the successive formation of three monolayers, followed by the formation of $3 \mathrm{D}$ phase which pumps the matter from the third layer. Does the decreasing part of the kinetic curves mean that the third layer is a non condensed (or overcritical) $2 \mathrm{D}$ phase which is smoothly compressed until the formation of $3 \mathrm{D}$ nuclei ? If not, the formation of 3D phase would be possible when the first patches of the condensed third layer appear and the kinetic curve would not have the same shape.

E. BAUER. - LEED indicates that a film which consists of 3 monolayers has the same lateral periodicity as a film consisting of 2 monolayers. Surface energy and strain energy are changing with the number of monolayers. I believe that this is the cause of the reported mode of film growth.

R. KERN. - You distinguished clearly 2 types of bonding in the mono and submonolayer range according to the electron affinity. In the case of attractive forces in the layer, $I$ think we can have real $2 \mathrm{D}$ condensed phases, but not in the case of repulsive forces. In the first case, the $2 \mathrm{D}$ phase must exist in an island form because the edge energies are $>0$ ( $2 \mathrm{D}$ equivalent of surface energy in $3 \mathrm{D}$ ), but in the second case the edge energies are $\leqslant 0$, this means in this last case that the atoms are ordered only if they are confined in a given space (surface). There is a nice old experiment of Renninger $(\sim 1954)$ showing that charged particles are able to form a long range order if these particles have a given surface available. A water solution of pentaerythridol is a bit evaporated. Pentaerythridol crystals are formed on the surface of the solution (density $<1$ ), and because these crystals have a polar character (pyroelectricity), the assembly of crystals gives a nice $2 D$ lattice. This lattice is only stable because the surface of the solution is laterely limited by the vessel. So I think that the 2D-phases with repulsive forces are not thermodynamically stable condensed phases but only mechanically stable condensed phases.

E. BAUER. - I agree with you. The mechanical constraints would here be potential barriers for surface diffusion. 